\title{
SOMA TÉRMICA DE ALGUMAS FASES DO CICLO DE DESENVOLVIMENTO DA ESCALA DE COUNCE PARA CULTIVARES SUL-BRASILEIRAS DE ARROZ IRRIGADO ( $\left.{ }^{1}\right)$
}

\author{
NEREU AUGUSTO STRECK $\left({ }^{2 *}\right)$; SIMONE MICHELON $\left({ }^{3}\right)$; LEOSANE CRISTINA BOSCO $\left({ }^{3}\right)$; \\ ISABEL LAGO $\left({ }^{3}\right)$; LIDIANE CRISTINE WALTER $\left({ }^{4}\right)$; HAMILTON TELLES ROSA $\left({ }^{5}\right)$; \\ GIZELLI MOIANO DE PAULA $\left({ }^{5}\right)$
}

\begin{abstract}
RESUMO
A escala de Counce é uma escala de desenvolvimento de arroz recente que vem sendo gradativamente adotada no Brasil. No entanto, a duração das fases em ${ }^{\circ} \mathrm{C}$ dia dessa escala ainda não está disponível para as cultivares brasileiras de arroz irrigado. Sendo assim, o objetivo deste trabalho foi determinar a soma térmica de algumas fases do ciclo de desenvolvimento da escala de CouncE para cultivares Sul-Brasileiras de arroz irrigado, utilizando-se diversos métodos de cálculo e diferentes temperaturas cardinais ao longo do ciclo de desenvolvimento. Um experimento a campo foi desenvolvido em Santa Maria, RS, durante dois anos agrícolas (2003/2004 e 2004/2005) e com cinco épocas de semeadura em cada um deles. $\mathrm{O}$ delineamento experimental foi o inteiramente casualizado com quatro repetições. Cada repetição consistia de um vaso de 12 litros com 10 plantas. As datas dos estágios de desenvolvimento R2 (aparecimento do colar da folha bandeira) e R9 (todos os grãos com casca marrom) da escala de CoUNCE foram registradas no colmo principal de cinco plantas por vaso. As cultivares utilizadas foram IRGA 421, IRGA 416, IRGA 417, IRGA 420, BR-IRGA 409, BRS 7 TAIM e EPAGRI 109. Considerando-se as temperaturas cardinais de $11{ }^{\circ} \mathrm{C}, 28{ }^{\circ} \mathrm{C}$ e $40{ }^{\circ} \mathrm{C}$, a soma térmica para completar a fase emergência-R2 foi de $700-800{ }^{\circ} \mathrm{C}$ dia na cultivar muito precoce, $1000-1400{ }^{\circ} \mathrm{C}$ dia nas cultivares precoces e $1300-1700$ ${ }^{\circ} \mathrm{C}$ dia nas cultivares médias e tardia, e a soma térmica para completar a fase R2-R9 foi de $400-700{ }^{\circ} \mathrm{C}$ dia e sem relação com o ciclo da cultivar.
\end{abstract}

Palavras-chave: temperatura, fenologia, estágios de desenvolvimento, graus-dia.

$\left({ }^{1}\right)$ Recebido para publicação em 27 de junho e aceito em 11 de dezembro de 2006.

$\left({ }^{2}\right)$ Departamento de Fitotecnia, Centro de Ciências Rurais (CCR), Universidade Federal de Santa Maria (UFSM), 97105-900 anta Maria (RS), Brasil. E-mail: nstreck1@smail.ufsm.br. Bolsista Produtividade em Pesquisa do Conselho de Desenvolvimento Científico e Tecnológico (CNPq). ( $\left.{ }^{*}\right)$ Autor correspondente.

$\left({ }^{3}\right)$ Estudante do Programa de Pós-Graduação em Agronomia, UFSM. Bolsista da Fundação Coordenação de Aperfeiçoamento de Pessoal de Nível Superior (CAPES).

$\left({ }^{4}\right)$ Estudante do curso de graduação em Agronomia, CCR, UFSM. Bolsista do Programa Institucional de Bolsas de Iniciação Científica do CNPq (PIBIC/CNPq/UFSM).

$\left({ }^{5}\right)$ Estudante do curso de graduação em Agronomia, CCR, UFSM. 


\section{ABSTRACT \\ THERMAL TIME OF SOME DEVELOPMENTAL PHASES OF THE COUNCE SCALE FOR IRRIGATED RICE CULTIVARS GROWN IN SOUTHERN BRAZIL}

The COUNCE scale is a developmental scale recently proposed for rice and that is being used in Brazil. However, the duration of developmental phases of this scale in degree-days is not available for Brazilian irrigated rice cultivars. Thus, the objective of this study was to determine the thermal time of some developmental phases of the Counce scale in irrigated rice cultivars grown in Southern Brazil using different calculation methods and cardinal temperatures throughout the crop cycle. A field experiment was carried out in Santa Maria, RS, Brazil, during two years (2003/2004 and 2004/2005), with five sowing dates in each growing season. The experimental design was completely randomized with four replications. Each replication was a 12-liter pot with 10 plants. The cultivars used were IRGA 421, IRGA 416, IRGA 417, IRGA 420, BR-IRGA 409, BRS 7 TAIM, and EPAGRI 109. The date of R2 (colar of the flag leaf visible) and R9 (all grains with brown hulls) stages of the COUNCE scale were recorded on the main culm of five tagged plants per pot. Using the cardinal temperatures of $11{ }^{\circ} \mathrm{C}, 28{ }^{\circ} \mathrm{C}$, and $40{ }^{\circ} \mathrm{C}$, the thermal time of the emergence-R2 phase was $700-800{ }^{\circ} \mathrm{C}$ day for the very early cultivar, $1000-1400{ }^{\circ} \mathrm{C}$ day for early cultivars and $1300-1700{ }^{\circ} \mathrm{C}$ day for intermediate and late cultivars, and the thermal time of the R2-R9 phase was $400-700{ }^{\circ} \mathrm{C}$ day with no relationship with the developmental cycle of the cultivar.

Key words: temperature, phenology, developmental stages, degree-days.

\section{INTRODUÇÃO}

O crescimento e o desenvolvimento vegetal são processos independentes que podem ocorrer simultaneamente ou não. Enquanto crescimento envolve aumento irreversível de dimensões físicas como área, comprimento, largura, altura, volume ou diâmetro de órgãos, desenvolvimento refere-se a processos que envolvem diferenciação celular, iniciação (morfogênese) e aparecimento de órgãos (Hodges, 1991; Streck et al., 2003). Um estágio de desenvolvimento é, geralmente, caracterizado pela data de aparecimento de um órgão na planta, enquanto o intervalo de tempo entre dois estágios de desenvolvimento pode ser chamado de fase de desenvolvimento (STRECK et al., 2003). A identificação dos estágios de desenvolvimento do arroz e a quantificação do tempo necessário para atingir cada estágio de desenvolvimento são importantes ferramentas que o orizicultor pode utilizar no planejamento e em práticas de manejo da lavoura orizícola (Alves et al., 2000; STEINMETZ et al., 2004; FreitAs et al., 2006).

A temperatura é o principal elemento meteorológico que afeta o desenvolvimento da planta de arroz (Oryza sativa L.) (Hodges, 1991; GAO et al., 1992; Sié et al., 1998; INFELD et al., 1998). A influência da temperatura nos processos de crescimento e desenvolvimento vegetal pode ser representada utilizando-se o conceito da soma térmica, com unidade ${ }^{\circ} \mathrm{C}$ dia, a qual é um melhor descritor de tempo biológico do que dias do calendário civil (GILMORE e Rogers, 1958; Russele et al., 1984; McMaster e SMiKa, 1988). Apesar de ser bastante utilizada, deve-se mencionar que o conceito da soma térmica está aberto a críticas (XUE et al., 2004). Entre as críticas está o fato de que existem vários métodos de cálculo (SANGOI e SiLva, 1986; StRecK et al., 2005), o que pode ser uma limitação para comparar os graus-dia de fases de desenvolvimento das cultivares em diferentes trabalhos. Algumas vezes também os relatos nos trabalhos não são claros quanto ao método de cálculo utilizado (McMaster e Wilhelm, 1997).

A maneira mais simples de cálculo da soma térmica é a que acumula os graus-dia acima de um valor crítico inferior (temperatura-base). Essa maneira de calcular é outra fonte de críticas, pois não é completamente realística do ponto de vista biológico, haja vista que a resposta dos processos biológicos à temperatura, inclusive desenvolvimento, é mais bem expressa em termos das três temperaturas cardinais, (temperatura mínima, ótima e máxima) (YIN et al., 1995; BONHOMME, 2000; STRECK, 2002). Outra fonte de crítica é com relação às temperaturas cardinais geralmente consideradas constantes ao longo do ciclo da cultura, muitas vezes, também, não é uma pressuposição adequada; essas temperaturas não são constantes durante o ciclo de desenvolvimento vegetal para muitas espécies (STRECK et al., 2003; SteinMETZ, 2004; Paula et al, 2005).

O ciclo de desenvolvimento do arroz tem sido frequentemente dividido em três fases (GAO et al, 1992; INFELD et al., 1998): a fase vegetativa que vai da emergência até a diferenciação da panícula; a fase reprodutiva que vai da diferenciação da panícula até a antese; e a fase de enchimento de grãos que vai da antese até a maturação fisiológica. Mais recentemente, Counce et al. (2000) propuseram dividir o ciclo de desenvolvimento do arroz em três fases utilizando critérios diferentes do que vinha sendo usado: a fase 
de plântula que vai da semeadura até a emergência; a fase vegetativa que vai da emergência até o aparecimento do colar da última folha (folha bandeira) no colmo principal e a fase reprodutiva que vai da diferenciação da panícula até a maturação fisiológica. Nesse caso, o fim da fase vegetativa e o início da fase reprodutiva se sobrepõem, pois a diferenciação da panícula ocorre durante a fase de emissão de folhas, quando ainda faltam, em média, aparecer três folhas no colmo principal (YIN e KROPFF, 1998). A escala de COUNCE vem sendo gradativamente adotada pelas Instituições de pesquisa em arroz como o IRGA (Instituto Rio Grandense do Arroz), a EMBRAPA (Empresa Brasileira de Pesquisa Agropecuária) e a EPAGRI (Empresa de Pesquisa Agropecuária e Extensão Rural de Santa Catarina). Por ser uma escala recente, a duração das fases de desenvolvimento desta escala está disponível apenas em dias do calendário civil para algumas cultivares brasileiras de arroz (FreITAS et al., 2006), mas não em graus-dia, o que constitui o incentivo para realizar este esforço científico. Oportuno também é calcular os graus-dia das fases de desenvolvimento dessa recente escala que está sendo adotada na cadeia produtiva do arroz por meio de diferentes métodos.

Diante do exposto, o objetivo deste trabalho foi determinar a soma térmica de algumas fases do ciclo de desenvolvimento da escala de CounCE et al. (2000) para cultivares sul-brasileiras de arroz irrigado, utilizando-se diversos métodos de cálculo e diferentes temperaturas cardinais ao longo do ciclo de desenvolvimento.

\section{MATERIAL E MÉTODOS}

Um experimento de campo foi desenvolvido durante dois anos agrícolas (2003/2004 e 2004/2005) na área experimental do Departamento de Fitotecnia da Universidade Federal de Santa Maria, Santa Maria (RS), Brasil (latitude: $29^{\circ} 43^{\prime} \mathrm{S}$, longitude: $53^{\circ} 42^{\prime} \mathrm{W}$ e altitude: $95 \mathrm{~m}$ ), localizada na região ecoclimática da Depressão Central do Rio Grande do Sul. O clima da região, segundo a classificação de Köppen, é Cfa, subtropical úmido sem estação seca definida com verões quentes (Moreno, 1961). O solo do local é uma transição entre a Unidade de Mapeamento São Pedro (Argissolo Vermelho distrófico arênico) e a Unidade de Mapeamento Santa Maria (Alissolo Hipocrômico argilúvico típico) (EMBRAPA, 1999).

As plantas de arroz foram cultivadas em vasos de $30 \mathrm{~cm}$ de diâmetro e $26 \mathrm{~cm}$ de altura com capacidade de 12 litros preenchidos com solo do local e enterrados, deixando-se uma borda de $5 \mathrm{~cm}$ acima do nível do solo. O espaçamento entre os vasos (de centro a centro dos vasos) foi de $1,5 \mathrm{~m} \times 0,8 \mathrm{~m}$. Foram realizadas cinco épocas de semeadura em cada ano agrícola. No ano agrícola $2003 / 2004$ as datas de semeadura foram $1 .^{\circ} / 9 / 2003,20 / 10 / 2003,21 / 11 /$ $2003,5 / 1 / 2004$ e $29 / 1 / 2004$ e no ano agrícola 2004 / 2005 as datas de semeadura foram $2 / 9 / 2004,7 / 10 /$ 2004, 4/11/2004, 3/12/2004 e 2/03/2005. Essas datas de semeadura foram selecionadas para se obterem semeaduras antes, durante e depois da época recomendada para a Região da Depressão Central do Rio Grande do Sul que é de $1 .^{\circ}$ de outubro a 10 de dezembro (SosBaI, 2005), e assim ter as plantas desenvolvendo-se em diferentes condições de temperatura. O delineamento experimental foi o inteiramente casualizado com quatro repetições. Cada repetição constituiu-se de um vaso com 10 plantas.

As cultivares utilizadas foram IRGA 421, IRGA 416, IRGA 417, IRGA 420, BR-IRGA 409, BRS 7 TAIM e EPAGRI 109, todas pertencentes à subespécie indica, tipo moderno, e recomendadas para o Rio Grande do Sul e Santa Catarina (SosbaI, 2005). Esses genótipos possuem variação considerável de ciclos, de muito precoce (IRGA 421) a tardio (EPAGRI 109) (SosbaI, 2005). Foram semeadas 30 sementes por vaso. O dia da emergência foi considerado quando $50 \%$ das plântulas estavam com o prófilo visível acima do nível do solo. O manejo das plantas foi feito com base nas recomendações técnicas para a cultura do arroz irrigado (Sosbai, 2005). A adubação foi realizada a partir da análise química do solo, antes da semeadura da primeira época. Usou-se $20 \mathrm{~g}$ vaso $^{-1}$ de adubo 711-9 na semeadura, e a adubação de cobertura de nitrogênio foi aplicada em duas vezes: a primeira no início do perfilhamento e a segunda na diferenciação da panícula ou ponto de algodão, usando-se $8,5 \mathrm{~g}$ vaso $^{-1}$ de uréia. A irrigação foi realizada deixandose uma lâmina de água de 5 a $7 \mathrm{~cm}$ nos vasos, a partir do estágio V3 da escala de Counce et al. (2000).

Uma semana após a emergência, cinco plantas por vaso foram marcadas com arames coloridos. No estágio V3 (CouncE et al., 2000), foi realizado um raleio das plantas, deixando-se 10 plantas por vaso. No colmo principal das plantas marcadas, observou-se a data de ocorrência dos estágios de desenvolvimento R2 (aparecimento do colar da folha bandeira) e R9 (maturação fisiológica: todas as espiguetas da panícula tinham os grãos com casca marrom) da escala de Counce et al. (2000). Para tanto, as plantas foram observadas diariamente durante o período experimental.

A soma térmica diária $\left(\mathrm{STd},{ }^{\circ} \mathrm{C}\right.$ dia) foi calculada por três métodos (GILMORE Junior e ROGERS, 1958; ARNOLD, 1960; STRECK, 2002): 
Método 1 (Gilmore Junior e Rogers, 1958; Arnold, 1960):

$$
\begin{aligned}
& \mathrm{STd}_{1}=(\text { Tmed-Tb).1dia, } \\
& \text { se Tmed }<\mathrm{Tb} \text { então Tmed }=\mathrm{Tb}
\end{aligned}
$$

Método 2 (Gilmore Junior e Rogers, 1958):

$\mathrm{STd}_{2}=($ Tmed $-\mathrm{Tb}) \cdot 1 \mathrm{dia}$,

se Tmed $<$ Tb então Tmed $=$ Tb e

se Tmed $>$ Tot então Tmed $=$ Tot

Método 3 (STRECK, 2002; STRECK et al., 2005):

$\mathrm{STd}_{3}=(\mathrm{Tmed}-\mathrm{Tb}) .1 \mathrm{dia}$

quando $\mathrm{Tb}=$ Tmed $=$ Tot $\mathrm{e}$

$\mathrm{STd}_{3}=($ Tot-Tb). $($ Tmax-Tmed $) /($ Tmax-Tot $)$

quando Tot $<$ Tmed $=$ Tmax,

se Tmed $<$ Tb então Tmed $=$ Tb e

se Tmed $>$ Tmax então Tmed $=$ Tmax

em que Tb, Tot, e Tmax são as temperaturas cardinais base, ótima e máxima de desenvolvimento do arroz e Tmed é a temperatura média diária do ar calculada pela média aritmética entre as temperaturas mínima e máxima diária do ar. A representação gráfica dos três métodos de cálculo da STd é apresentada na Figura 1. A soma térmica acumulada $\left(\mathrm{STa},{ }^{\circ} \mathrm{C}\right.$ dia) a partir da emergência para cada método foi calculada por $\mathrm{STa}_{1}=\sum \mathrm{STd}_{1}, \mathrm{STa}_{2}=\sum \mathrm{STd}_{2}, \mathrm{STa}_{3}=\sum \mathrm{STd}_{3}$, respectivamente para os métodos 1,2 e 3 .

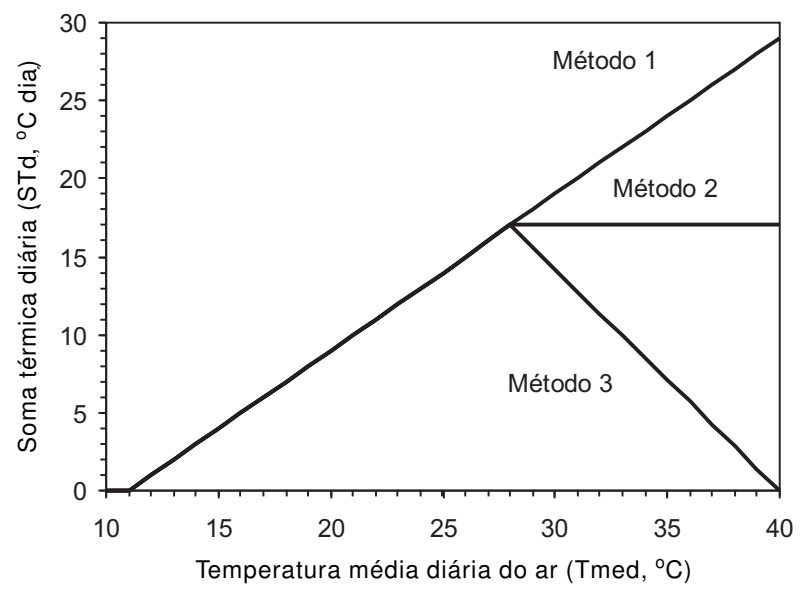

Figura 1. Representação gráfica dos três métodos de cálculo da soma térmica diária (Método 1, Método 2, Método 3) usados no estudo. As temperaturas cardinais ( $\mathrm{Tb}$, Tot e Tmax) são da fase de emergência ao aparecimento do colar da folha bandeira (EM-R2): 11, 28 e $40{ }^{\circ} \mathrm{C}$. Santa Maria (RS), Brasil, 2003-2005.
As temperaturas mínima e máxima diária do ar foram medidas em uma estação meteorológica convencional, pertencente ao $8 .^{\circ}$ Distrito de Meteorologia/Instituto Nacional de Meteorologia, localizada a aproximadamente $200 \mathrm{~m}$ da área experimental. Com relação às temperaturas cardinais, consideraram-se duas situações. Primeira, que as temperaturas cardinais são constantes ao longo do ciclo de desenvolvimento, já que essa é uma pressuposição usada em modelos de arroz (GAO et al., 1992). Nesse caso, assumiu-se $\mathrm{Tb}=11^{\circ} \mathrm{C}$ (INFELD et al., 1998), $\mathrm{Tot}=28^{\circ} \mathrm{C}$ (Steinmetz, 2004) e Tmax $=40^{\circ} \mathrm{C}$ (GAO et al., 1992). Segunda, considerou-se que as temperaturas mudam ao longo do ciclo de desenvolvimento. No segundo caso, dividiu-se o ciclo de desenvolvimento do arroz em duas fases: EM-R2 e R2-R9. Optou-se por dividir o ciclo nessas duas fases, pois a primeira fase (EM-R2) se refere à emissão de folhas, enquanto a segunda (R2-R9), caracteriza-se pelo aparecimento das estruturas reprodutivas e pelo enchimento de grãos, todos os estágios facilmente identificáveis no campo, sem a necessidade de destruir a planta. As temperaturas cardinais $(\mathrm{Tb}$, Tot e Tmax) na fase EM-R2 foram 11,28 e $40{ }^{\circ} \mathrm{C}$ e na fase R2-R9 foram de 15,25 e $30^{\circ} \mathrm{C}$. Assumiu-se que uma $\mathrm{Tb}$ de $15^{\circ} \mathrm{C}$ na fase R2-R9 é mais realística do que 11 ${ }^{\circ} \mathrm{C}$, pois o arroz é sensível à temperatura igual ou inferior a $15{ }^{\circ} \mathrm{C}$ durante a microsporogênese e embriogênese (Buriol et al., 1991; STEINMETZ, 2004; SOSBAI, 2005), que ocorrem durante o emborrachamento e durante o aparecimento do colar da folha bandeira, ou seja, próximo a R2 (Counce et al., 2000). A diminuição de Tot e Tmax na fase R2-R9 em relação à fase EM-R2 foi devido ao fato de que a fase do arroz mais sensível às altas temperaturas é a floração, e o enchimento de grãos é mais eficiente quando as temperaturas não são tão elevadas (STEINMETZ, 2004).

A duração das fases EM-R2 e R2-R9 também foi medida em dias do calendário civil, para comparação com os métodos de soma térmica. A estatística utilizada para comparar a eficiência da soma térmica como descritor de tempo em comparação com dias do calendário civil foi o coeficiente de variação $(\mathrm{CV}, \%)$ entre as épocas de semeadura nos dois anos agrícolas (PAULA et al., 2005). Quanto menor é o CV melhor o descritor de tempo biológico.

\section{RESULTADOS E DISCUSSÃO}

A duração do ciclo total (EM-R9) das cultivares de arroz irrigado expressas em dias do calendário civil e em ${ }^{\circ} \mathrm{C}$ dia pelos diferentes métodos de soma térmica, com temperaturas cardinais constantes e variáveis ao longo do ciclo, estão representadas na Figura 2. Comparando as cultivares, nota-se a grande 
diferença de ciclo entre a cultivar muito precoce (IRGA 421) e a tardia (EPAGRI 109). As cultivares BR-IRGA 409 e BRS 7 TAIM são de ciclo médio, enquanto IRGA 420, IRGA 417 e IRGA 416 são de ciclo precoce (SosBAI, 2005). Entre os métodos de cálculo da soma térmica, os graus-dia diminuíram do método $1\left(\mathrm{STa}_{1}\right)$ para o método $3\left(\mathrm{STa}_{3}\right)$ e a diferença entre $\mathrm{STa}_{1}$ e $\mathrm{STa}_{3}$ foi maior quando as temperaturas cardinais foram diferentes ao longo do ciclo da cultura. Esse decréscimo de graus-dia do método 1 para o método 3 ocorreu em função da Tmed, durante o ciclo de desenvolvimento, ter sido em vários dias superior à Tot, o que acarreta diminuição de STa do método 1 para o método 3 (Figura 1). Diferentes valores da soma térmica em função do método de cálculo foram relatados em trabalhos anteriores com outras espécies, como girassol, trigo e milho (SANGOI e SILVA, 1986; McMaster e Wilhelm, 1997). Portanto, é importante que se descreva detalhadamente o método utilizado no cálculo dos graus-dia, o que nem sempre se verifica nos trabalhos científicos (MCMAster e WiLHeLm, 1997), tornando, às vezes, difícil a comparação de resultados entre diferentes autores e levando a erros na simulação de processos e eventos biológicos com base na soma térmica.

Para um mesmo método de cálculo, a soma térmica foi maior quando as temperaturas cardinais de desenvolvimento foram consideradas constantes ao longo do ciclo do que quando foram diferentes (Figura 2). A menor soma térmica, quando as temperaturas cardinais foram diferentes, deve-se em parte ao fato de que a mudança nas temperaturas cardinais durante a fase R2-R9 ocasionou diminuição da diferença entre $\mathrm{Tb}$ e Tot, que é o máximo de acúmulo térmico diário possível, e em parte devido à Tmed ter sido freqüentemente maior que a temperatura ótima de 25 ${ }^{\circ} \mathrm{C}$ durante essa fase. Os coeficientes de variação $(\mathrm{CV}$, $\%$ ) da duração do ciclo total de desenvolvimento (EMR9) em dias do calendário civil e em soma térmica calculada por diferentes métodos e com temperaturas cardinais constantes e variáveis ao longo do ciclo da cultura são apresentados na Figura 3. Quando se consideraram as temperaturas cardinais constantes durante o ciclo de desenvolvimento do arroz, o CV foi ligeiramente menor com a soma térmica calculada pelo método $3\left(\mathrm{STa}_{3}\right)$, seguidas por $\mathrm{STa}_{2}, \mathrm{STa}_{1}$ e dias após a emergência. Esses resultados confirmam que o efeito da temperatura sobre o desenvolvimento vegetal é mais bem representado levando-se em conta as três temperaturas cardinais (YIN et al., 1995; STRECK, 2002; StRECK et al., 2003), o que é contemplado no método 3 , embora a pequena diferença de $\mathrm{CV}$ entre os métodos (Figura 3) possa ser motivo para se propor o uso do método mais simples de cálculo $\left(\mathrm{STa}_{1}\right)$, o qual apenas leva em conta o acúmulo térmico acima da $\mathrm{Tb}$ (eq. 1).
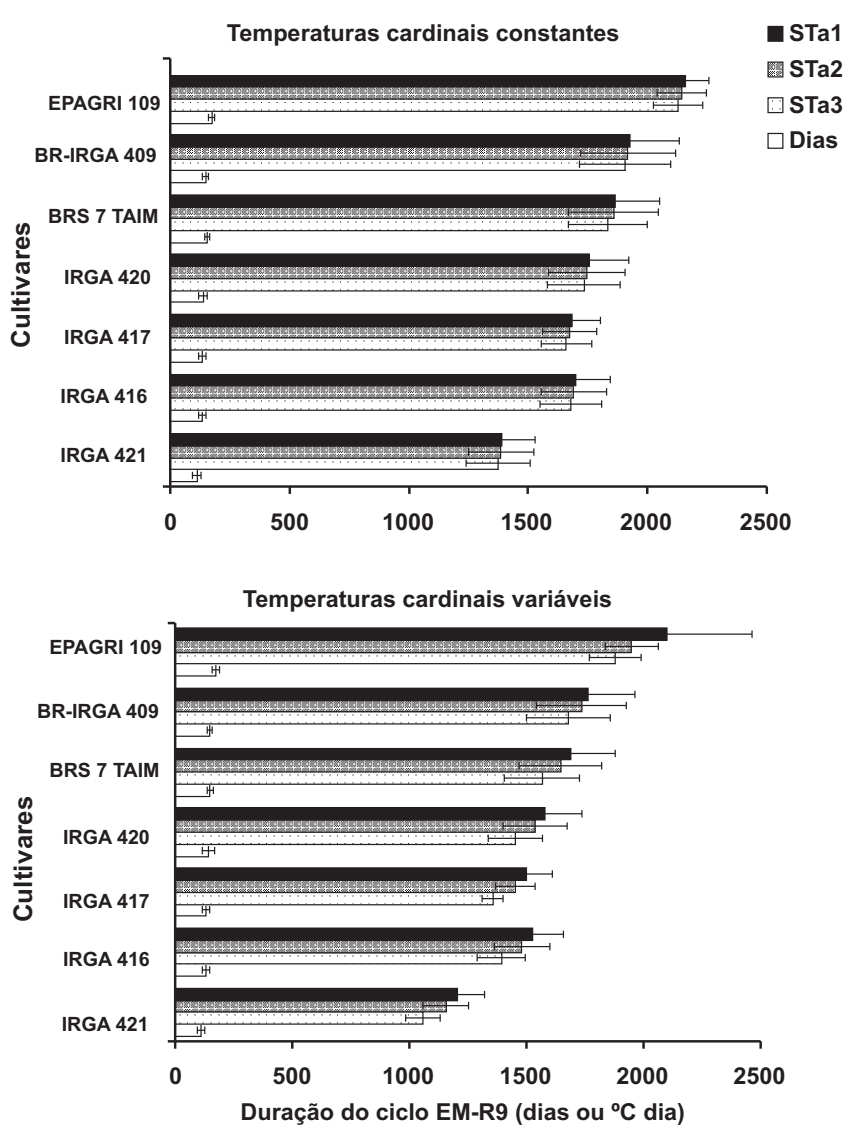

Figura 2. Duração em dias do calendário civil e em soma térmica acumulada $\left({ }^{\circ} \mathrm{C}\right.$ dia) do ciclo total (EM-R9) de cultivares de arroz irrigado. A soma térmica foi calculada por três métodos de cálculo $\left(\mathrm{STa}_{1}, \mathrm{STa}_{2}, \mathrm{STa}_{3}\right)$ considerando-se as temperaturas cardinais constantes e variáveis ao longo do ciclo de desenvolvimento. As barras representam a média e o desvio padrão de cinco épocas de semeadura nos anos agrícolas 2003/2004 e 2004/2005. Santa Maria (RS), Brasil.

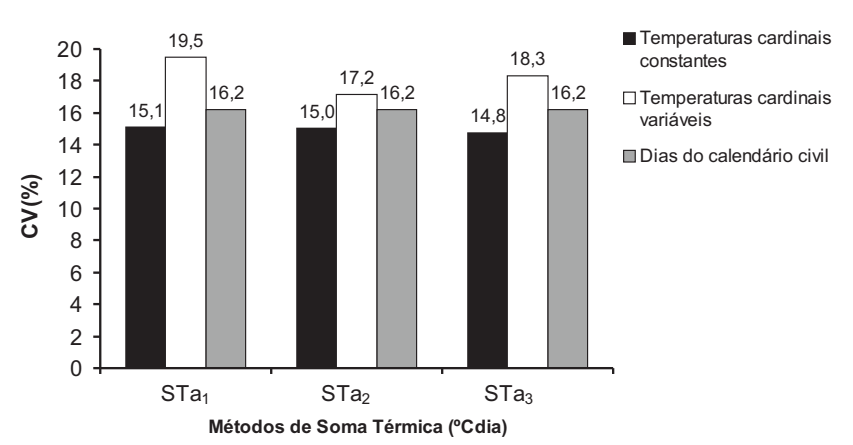

Figura 3. Coeficiente de variação (CV\%) para os três métodos de cálculo de soma térmica $\left(\mathrm{STa}_{1}, \mathrm{STa}_{2}, \mathrm{STa}_{3}\right)$, com temperaturas cardinais constantes e variáveis, e para dias do calendário civil, da duração do ciclo de desenvolvimento EM-R9 de cultivares de arroz irrigado nos anos agrícolas 2003/2004 e 2004/2005. Santa Maria (RS), Brasil. EM=Emergência, R9=Maturação fisiológica. 
Quando as temperaturas cardinais foram diferentes na fase R2-R9, o CV foi ligeiramente menor com o método $\mathrm{STa}_{2}$, mas todos foram maiores que os dias do calendário civil e os métodos de soma térmica considerando as temperaturas cardinais constantes (Figura 3). Esses resultados são surpreendentes, pois, esperava-se que uma maior $\mathrm{Tb}$ e menor Tot e Tmax no fim do ciclo de desenvolvimento resultaria em CV menor, pois essa pressuposição parece mais realística do ponto de vista biológico (STEINMETZ, 2004).

As durações, em ${ }^{\circ} \mathrm{C}$ dia, das fases de desenvolvimento do arroz EM-R2 e R2-R9 da escala de Counce et al. (2000), nos dois anos agrícolas, estão representadas na figura 4 . A soma térmica usada na figura 4 foi calculada pelo método $3\left(\mathrm{STa}_{3}\right)$ com temperaturas cardinais constantes, pois essa combinação resultou em menor CV (Figura 3). A duração do ciclo total de desenvolvimento e das fases variou entre as cultivares, entre épocas de semeadura e entre os anos. A fase mais longa foi a fase EM-R2 perfazendo de $55 \%$ a $80 \%$ da duração do ciclo total das cultivares (Figura 4). Quanto mais longo o ciclo total da cultivar, maior a fração da duração da fase EM-R2 em relação ao ciclo total. Por exemplo, para a cultivar IRGA 421 (ciclo muito precoce), a duração da fase EM-R2 foi em torno de $55 \%$, enquanto para a cultivar EPAGRI 109 (ciclo tardio), a fase EM-R2 representou em torno de $80 \%$ do ciclo total. A cultivar muito precoce (IRGA 421) completou a fase EM-R2 entre 700 e $800{ }^{\circ} \mathrm{C}$ dia nos dois anos; para as cultivares precoces (IRGA 416, IRGA 417 e IRGA 420) foram necessários de 1.000 a $1.400{ }^{\circ} \mathrm{C}$ dia, e para as cultivares médias e tardias (BR-IRGA 409, BRS 7 TAIM, EPAGRI 109) foram necessários de 1.300 a $1.700{ }^{\circ} \mathrm{C}$ dia nessa fase. INFELD et al. (1998) também observaram diferenças na duração da fase vegetativa (emergência até a diferenciação da panícula) para diferentes grupos de cultivares sul-brasileiras de arroz, cujas cultivares precoces necessitaram de $536{ }^{\circ} \mathrm{C}$ dia, as cultivares de ciclo médio, $638^{\circ} \mathrm{C}$ dia e as tardias, de $772{ }^{\circ} \mathrm{C}$ dia. A fase R2-R9 variou bem menos entre as cultivares, ficando entre 400 e $700{ }^{\circ} \mathrm{C}$ dia e sem relação com o ciclo da cultivar.

Em relação às épocas de semeadura, as duas últimas datas de semeadura nos dois anos agrícolas tiveram menor duração das fases de desenvolvimento (Figura 4). Essas duas datas de semeadura tardias foram feitas fora da época recomendada para o arroz irrigado na Região da Santa Maria (SosbaI, 2005), mas são interessantes neste estudo, pois representam condições térmicas durante o ciclo de desenvolvimento diferentes daquelas recomendadas para a cultura. Na última data de semeadura do ano agrícola 2004/2005, nenhuma cultivar atingiu o estágio R9, devido às baixas temperaturas em junho de 2005. A redução da duração das fases de desenvolvimento nas últimas duas épocas de semeadura pode estar associada a alguma resposta fotoperiódica dos genótipos. $\mathrm{O}$ arroz é uma planta de dia curto (Vergara et al., 1969; Yin et al., 1997; Yin e KROPFF, 1998), de modo que o fotoperíodo decrescente, a partir de fevereiro, pode ter acelerado o desenvolvimento das cultivares.
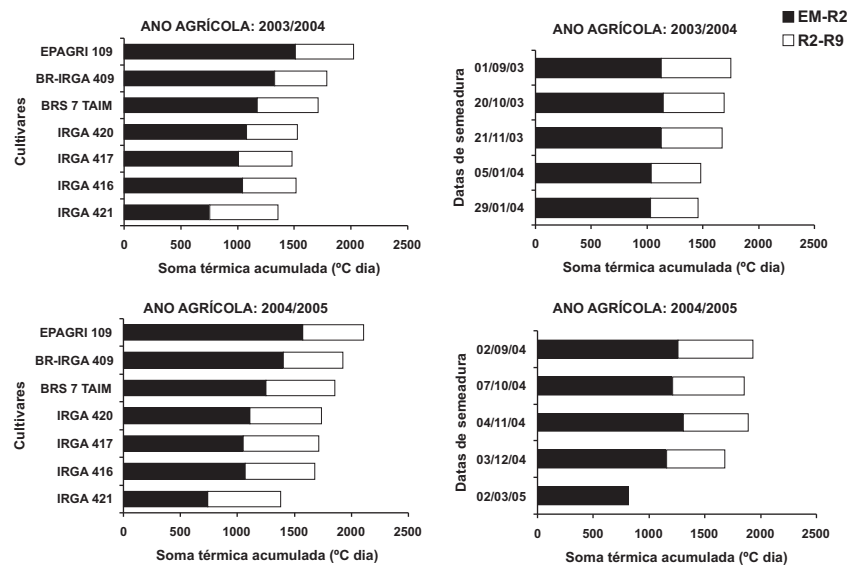

Figura 4. Soma térmica acumulada $\left({ }^{\circ} \mathrm{C}\right.$ dia $)$ pelo método $3\left(\mathrm{STa}_{3}\right)$ (média para as cultivares e para épocas de semeadura), das fases de EM-R2 e R2-R9 do ciclo de desenvolvimento de cultivares de arroz irrigado em várias épocas de semeadura para os anos agrícolas de 2003/ 2004 e 2004/2005. Na soma térmica das cultivares (painéis da esquerda) consideraram-se as quatro primeiras épocas de semeadura, pois algumas cultivares não completaram o ciclo na quinta época do ano agrícola 2004/2005. Santa Maria (RS), Brasil.

Outra possível causa da diminuição da soma térmica, nas últimas duas datas de semeadura, pode ser a linearidade entre a temperatura e o desenvolvimento vegetal assumidos no cálculo, o que não é totalmente realístico do ponto de vista biológico, já que a resposta de processos biológicos à temperatura é linear apenas em uma parte e não em toda a faixa de variação entre as temperaturas cardinais (Streck, 2002; Streck et al., 2003; StReCK, $2004 a, b)$. Próximo às temperaturas cardinais, a resposta não linear do desenvolvimento à temperatura introduz erros no cálculo da soma térmica (STRECK, 2002). Nas últimas duas datas de semeadura, as temperaturas do ar ficaram mais freqüentemente próximas à $\mathrm{Tb}$. Se a temperatura do ar é pouco menor que a $\mathrm{Tb}$ (por exemplo, $1^{\circ} \mathrm{C}$ ) não se acumula nenhum ${ }^{\circ} \mathrm{C}$ dia, mas existe um pequeno desenvolvimento da planta (STRECK, 2002), o que se ocorrer durante vários dias a leva a avançar no seu desenvolvimento, embora não haja cômputo de graus-dia pelos métodos usados. 
Outras causas da variação da soma térmica entre épocas de semeadura e anos podem estar ligadas a outras pressuposições envolvidas no seu cálculo; por exemplo, a região de desenvolvimento (ápice meristemático) tem a mesma temperatura que a temperatura média diária do ar, medida na estação meteorológica, e a temperatura diária não permanece abaixo da $\mathrm{Tb}$ ou acima da Tmax por muito tempo (McMaster e Wilhelm, 1997). Essas pressuposições, não necessariamente, são válidas em todos os dias durante o ciclo de desenvolvimento da cultura.

No entanto, considerando-se as três primeiras épocas de semeadura, a variação da soma térmica nas fases é menor (Figura 4). Essas épocas estão mais próximas da época recomendada para a cultura na Região de Santa Maria. FreitAs et al. (2006) caracterizaram algumas fases de desenvolvimento da escala de Counce et al. (2000) para três cultivares de arroz irrigado (IRGA 421, IRGA 417, BR-IRGA 419), usando dias do calendário civil como unidade de tempo, em três datas de semeadura (14/10/03, 13/11/03 e 18/ 12/03) em experimento realizado próximo a Porto Alegre (RS), localizado como Santa Maria também na Região ecoclimática da Depressão Central do Rio Grande do Sul. Ambos obtiveram variação acentuada na duração das fases, que diminuíram com o atraso da semeadura. Recomenda-se, portanto, que a duração das fases seja expressa em ${ }^{\circ} \mathrm{C}$ dia ao invés de dias do calendário civil.

\section{CONCLUSÕES}

1. Considerando-se as temperaturas cardinais de $11^{\circ} \mathrm{C}, 28^{\circ} \mathrm{C}$ e $40^{\circ} \mathrm{C}$, a soma térmica para completar a fase EM-R2 foi de $700-800{ }^{\circ} \mathrm{C}$ dia na cultivar muito precoce, $1.000-1.400{ }^{\circ} \mathrm{C}$ dia nas cultivares precoces e 1.300-1.700 ${ }^{\circ} \mathrm{C}$ dia nas cultivares médias e tardias, e a soma térmica para completar a fase R2-R9 foi de 400$700{ }^{\circ} \mathrm{C}$ dia e sem relação com o ciclo da cultivar.

2. A fase EM-R2 perfaz $55 \%-80 \%$ do ciclo total (EM-R9), com cultivares de ciclo mais longo tendo maior fração da duração da fase EM-R2.

\section{AGRADECIMENTOS}

Ao Instituto Rio Grandense do Arroz (IRGA) pela ajuda financeira no primeiro ano e pelo fornecimento das sementes nos dois anos deste estudo.

\section{REFERÊNCIAS}

ALVES, V.C.; PEDRO JÚNIOR, M.J.; SENTELHAS, P.C.; AZZINI, L.E. Exigências térmicas do arroz irrigado 'IAC 4440'. Revista Brasileira de Agrometeorologia, Santa Maria, v.8, n.2, p.171-174, 2000.
ARNOLD, C.Y. Maximum-minimum temperatures as a basis for computing heat units. Journal of the American Society for Horticultural Sciences, Alexandria, v. 76, p. 682 - 692, 1960.

BONHOMME, R. Bases and limits to using 'degree.day' units. European Journal of Agronomy, Amsterdam, v.13, n.1, p.110, 2000.

BURIOL, G.A.; ESTEFANEL, V.; SCHNEIDER, F.M.; HELDWEIN, A.B. Ocorrência e duração das temperaturas mínimas diárias do ar prejudiciais à fecundação das flores do arroz em Santa Maria, RS. 1- Probabilidades de ocorrência. Ciência Rural, Santa Maria, v. 21, n. 1, p. 23-34, 1991.

COUNCE, P.; KEISLING, T.C.; MITCHELL, A.J. A uniform, objective, and adaptive system for expressing rice development. Crop Science, Madison, v.40, n.2, p. 436-443, 2000.

EMBRAPA. Sistema Brasileiro de Classificação de Solos. Brasília: Embrapa-SPI; Embrapa-CNPS, 1999. 412p.

FREITAS, T.F.S.; SILVA, P.R.F.; STRIEDER, M.L.; SILVA, A.A. Validação de escala de desenvolvimento para cultivares brasileiras de arroz irrigado. Ciência Rural, Santa Maria, v.36, n.2, p. 404-410, 2006.

GAO, L.; JUN, Z.; HUANG, L. Rice clock model - a computer model to simulate rice development. Agricultural and Forest Meteorology, Amsterdan, v. 60, n. 1, p. 1 - 16, 1992.

GILMORE JUNIOR., E.C.; ROGERS, J. S. Heat units as a method of measuring maturity in corn. Agronomy Journal, Madison, v.50, n.10, p.611-615, 1958.

HODGES, T. Predicting crop phenology. Boca Raton: CRC, 1991. 233 p.

INFELD, J. A.; SILVA, J. B.; ASSIS, F. N. Temperatura base e graus-dia durante o período vegetativo de três grupos de cultivares de arroz irrigado. Revista Brasileira de Agrometeorologia, Santa Maria, v. 6, n.2, p. 187-191, 1998.

McMASTER,G.S.; SMIKA, D.E. Estimation and evaluation of winter wheat phenology in the Central Great Plains. Agricultural and Forest Meteorology, Amsterdam, v.43, n.1, p.1-18, 1988 .

McMASTER, G.S.; WILHELM, W.W. Growing degree-days: one equation, two interpretations. Agricultural and Forest Meteorology, Amsterdam, v.87, p.291-300, 1997.

MORENO, J. A. Clima do Rio Grande do Sul. Porto Alegre: Secretaria da Agricultura, 1961. 46p.

PAULA, F.L.M.; STRECK, N.A; HELDWEIN, A.B.; BISOGNIN, D.A; PAULA, A.L.; DELLAI, J. Soma térmica de algumas fases do ciclo de desenvolvimento da batata (Solanum tuberosum L.). Ciência Rural, Santa Maria, v. 35, n. 5, p. 1034-1042, 2005.

RUSSELE, M.P.; WILHELM, W. W.; OLSON, R.A.; POWER, J.F. Growth analysis based on degree days. Crop Science, Madison, v. 24, n.1, p. 28-32, 1984. 
SANGOI, L.; SILVA, P.R.F. Comparação entre métodos de cálculo de unidades térmicas e os dias do calendário na previsão de subperíodos de desenvolvimento do girassol. Pesquisa Agropecuária Brasileira, Brasília, v.21, p. 901-908, 1986.

SIÉ, M.; DINGKUHN, M.; WOPEREIS, M.C.S.; MIEZAN, K.M. Rice crop duration and leaf appearance rate in a variable thermal environment. I. Development of an empirically based model. Field Crops Research, Amsterdam, v. 57, n. 1, p. 1- 13, 1998.

SOSBAI (Sociedade Sul Brasileira de Arroz irrigado). Arroz irrigado: recomendações técnicas da pesquisa para o Sul do Brasil. Santa Maria: SOSBAI, 2005.159p.

STEINMETZ, S. Influência do clima na cultura do arroz irrigado no Rio Grande do Sul. In: GOMES, A.S.; MAGALHÃES JR, A. M. Arroz irrigado no Sul do Brasil. Brasília: Embrapa, 2004. p. $45-74$.

STEINMETZ, S.; INFELD, J.A.; ASSIS, F.N.; WREGE, M.S.; FERREIRA, J.S.A. Uso do método de graus-dia para estimar a data de diferenciação da panícula de grupos de cultivares de arroz irrigado no Rio Grande do Sul. Pelotas: Embrapa Clima Temperado, 2004. $14 \mathrm{p}$.

STRECK, N.A. A generalized nonlinear air temperature response function for node appearance rate in muskmelon (Cucumis melo L.). Revista Brasileira de Agrometeorologia, Santa Maria, v.10, n.1, p.105-111, 2002.

STRECK, N.A.; WEISS, A.; XUE, Q.; BAENZIGER, P.S. Improving predictions of developmental stages in winter wheat: A modified Wang and Engel model. Agricultural and Forest Meteorology, Amsterdam, v. 115, n 3-4, p.139-150, 2003.

STRECK, N.A. A temperature response function for development of the crysanthemun (Chrysantemun $x$ morifolium Ramat.). Ciência Rural, Santa Maria, v.34, n.1, p.49-54, 2004a.
STRECK, N.A. A temperature response function for modeling leaf growth and development of the African violet (Saintpaulia ionantha Wendl). Ciência Rural, Santa Maria, v.34, n.1, p.55-62, $2004 b$.

STRECK, N.A.; TIBOLA, T.; LAGO, I.; BURIOL, G.A.; HELDWEIN, A.B.; SCHNEIDER, F.M.; ZAGO, V. Estimativa do plastocrono em meloeiro (Cucumis melo L.) cultivado em estufa plástica em diferentes épocas do ano. Ciência Rural, Santa Maria, v.35, n.6, p.1275-1280, 2005.

VERGARA, B.S.,CHANG,T.T., LILIS,R.. The flowering response of the rice plant to photoperiod: A review of the literature. Los Baños: International Rice Research Institute, 1969. 28 p. (Technical Bulletin, 8)

XUE, Q.; WEISS, A.; BAENZIGER, P.S. Predicting leaf appearance in field grown winter wheat: evaluating linear and non-linear models. Ecological Modelling, v. 175, p.261270, 2004.

YIN, X.; KROPFF, M.J.; MCLAREN, G.; VISPERAS, R.M. A nonlinear model for crop development as a function of temperature. Agricultural and Forest Meteorology, Amsterdam, v.77. p. 1-16, 1995.

YIN, X.; KROPFF, M.; YNALVEZ, M.A. Photoperiodically sensitive and insensitive phases of preflowering development in rice. Crop Science, Madison, v. 37, p.182-190, 1997.

YIN, X.; KROPFF, M. The effect of photoperiod on interval between panicle initiation and flowering in rice. Field Crops Research, Amsterdam, v. 57, p. 301- 307, 1998. 